\title{
Editorial overview: Social insects: The internal rules of ant societies
}

\section{Nathalie Stroeymeyt and Laurent Keller}

Current Opinion in Insect Science 2014, 5:iv-v

For a complete overview see the Issue

Available online 23rd October 2014

http://dx.doi.org/10.1016/j.cois.2014.10.002

2214-5745/C 2014 Elsevier Inc. All rights reserved.

\section{Nathalie Stroeymeyt and Laurent Keller}

Department of Ecology and Evolution, Biophore, UNIL-Sorge, University of Lausanne, CH-1015 Lausanne, Switzerland e-mail: Nathalie.Stroeymeyt@unil.ch

Nathalie Stroeymeyt is a post-doctoral researcher in Professor Laurent Keller's group at the University of Lausanne, Switzerland. Her primary research interests are focused on collective organisation, decision-making and social homeostasis in animal societies, using ants as a model system.

Laurent Keller is professor of evolutionary biology and head of the Department of Ecology and Evolution at the University of Lausanne. He uses ants as model organism to study the principles governing the origin and evolution of animal societies. He has been particularly interested in how interactions between genetic and social factors jointly influence individual behaviour and colony social organisation.
The evolution of eusociality, defined as the emergence of societies with reproductive division of labour, overlapping generations, and cooperative care of young, is considered one of the major transitions in evolution and the basis of the tremendous ecological success of social insects (ants, some bees and wasps, termites) $[1,2]$. However, the organisation of individuals into highly cooperative societies requires the existence of intricate regulation mechanisms to ensure successful growth, survival and reproduction of the colony. The reviews in this issue present recent advances on several such mechanisms, and highlight multiple levels of complexity in the organisation and functioning of social insect colonies.

The coordination of activities within social insect colonies requires frequent interactions among group members, for example to transfer information or to accomplish a common task. This makes social insects highly vulnerable to disease, because the high density of closely-related individuals provides ideal conditions for pathogen transmission [3]. In the first review, Stroeymeyt and colleagues consider how interaction patterns within social insect colonies may be organised so as to hinder pathogen transmission within social insect colonies, a process known as organisational immunity. They highlight recent advances in data collection techniques and analysis methods which provided new tools to formally investigate the effects of interaction network structure on disease spread. An important message is that heterogeneities in the distribution of interactions within colonies may be a crucial factor in mitigating disease threat.

Heterogeneities in interactions are a direct consequence of the great diversity of individual behaviour characterising most social insect colonies. The second review, by Le Boeuf and Grozinger, explores behavioural variation and its possible underlying molecular mechanisms in further detail. They consider the complex interplay between individual and group behavioural variation, describing both how individuals influence the behaviour of the whole colony, and how colony composition in turn determines individual behaviour. The behavioural flexibility displayed at both individual and collective levels allows colonies to adjust to environmental changes and maintain their function in a broad range in conditions.

Behavioural variation among individuals within a colony is often accompanied by differences in physiology and metabolism [1]. These phenotypic differences may lead to specific nutritional needs. Variation in nutritional needs may be even more pronounced between individuals at varying developmental stages (e.g. brood versus adults) and between individuals belonging to morphologically distinct castes (e.g. queen versus workers). In 
the third review, Feldhaar describes how ant colonies meet the challenge of fulfilling the nutritional needs of all colony members. This requires complex regulation of both foraging and food redistribution among individuals. She then introduces the concept of nutritional niche plasticity, that is, the extent to which colonies can deviate from their nutritional optimum without incurring fitness costs. Feldhaar highlights that nutritional niche plasticity may have important ecological consequences, and calls for further studies investigating the link between nutrition and ant community structure.

The evolution of behavioural, physiological and morphological differentiation among individuals in insect societies has led to specific adaptations. For example, queens, who are critical in ensuring colony survival and reproduction, usually have a much longer lifespan than workers [1]. Theory predicts that longer lifespan is achieved by organisms investing more in somatic maintenance, thus delaying the effect of senescence through accumulation of somatic damage. In the fourth review, Lucas and Keller examine the evidence for caste differences in somatic damage accumulation and investment in somatic maintenance. Surprisingly, the available data provide no clear support that the longer-lived castes invest more in repair mechanisms than shorter-lived individuals. They conclude that somatic maintenance may not be the main mechanism through which queens prolong their lifespan, and suggest possible alternatives.

In the final review, Robinson considers a yet higher level of organisation than the colony. In some species, colonies are distributed over several spatially distinct, but socially interconnected nests. This system of social organisation, known as polydomy, is often associated with great ecological success. Robinson describes the functioning of multiple-nest colonies and examines the benefits and costs of this type of social organisation. Interestingly, individual nests within complex polydomous systems appear to be organised according to similar principles as those underlying the organisation of individual insects within single-nest colonies (as described in the first four reviews). For example, polydomous colonies show division of labour between nests specialising in different tasks, just as there is division of labour between specialised workers within each nest (Le Boeuf and Grozinger). Food appears to be redistributed across nests so that each one fulfils their nutritional needs, mirroring the redistribution of food among individuals within nests (Feldhaar). Robinson also highlights that the network of interconnecting trails may be structured so as to decrease the risk of disease transmission between nests, a phenomenon reminiscent of within-nest organisational immunity (Stroeymeyt et al.). Finally, nests vary in their lifespan just as individuals do within each nest (Lucas and Keller). These striking similarities between the organising principles and challenges at two different levels of complexity (groups of individuals versus groups of nests) suggest that social insects may have evolved sociality twice, first by forming societies of individuals and later societies of societies.

\section{References}

1. Hölldobler B, Wilson EO: The Ants. Cambridge, MA, USA: Harvard University Press; 1990.

2. Maynard Smith J, Szathmáry E: The Major Transitions in Evolution. Oxford University Press; 1995.

3. Cremer S, Armitage SAO, Schmid-Hempel P: Social immunity. Curr Biol 2007, 17:R693-R702. 